\title{
Radiofrequency ablation as a concomitant procedure for the treatment of atrial fibrillation during cardiac surgery
}

\author{
Tiwari KK', 2, 3, Gasbarri T², Bevilacqua S3, Jawarkar $\mathrm{M}^{3}$, Shah $\mathrm{M}^{3}$, Doshi $\mathrm{C}^{3}$, Glauber $\mathrm{M}^{4}$ \\ ${ }^{1}$ Kaushal K Tiwari, Associate Professor, Head of Department, Department of CTVS, College of Medical Sciences, Bharatpur, \\ Chitwan, Nepal; ${ }^{2}$ Tommaso Gasbarri, Consultant Cardiac Surgeon, Department of Adult Cardiac Surgery, G. Paquinucci Heart \\ Hospital, Fondazione Toscana G. Monasterio, Via Aurelia Sud, Massa, 54100, Italy; ${ }^{3}$ Stefano Bevilacqua, ${ }^{3}$ Manish Jawarkar, \\ ${ }^{3}$ Mausam Shah, ${ }^{3}$ Chirag Doshi, Cardiac Surgeon, Department of CVTS, UN Mehta Institute of Cardiology and Research \\ Center, Ahmedabad, Gujarat, India; ${ }^{4}$ Mattia Glauber, Professor, Head of the Department, Department of Cardiovascular \\ Surgery, Instituto clinic Sant Ambrogio, Gruppo ospedaliero San Donato, Milan, Italy.
}

\begin{abstract}
Atrial fibrillation is the most common type of arrhythmia with increasing burden for stroke and thromboembolic events. Medical treatment of atrial fibrillation hasn't shown promising results, so alternative method of treatment is emerging out. Cox-Maze procedure has been used for decades for the treatment of atrial fibrillation. Surgical treatment of atrial fibrillation with traditional Cox-Maze procedure is a complex and technically challenging procedure limiting its use in clinical practice. Recently, radiofrequency ablation is being used as a modification of Cox-Maze procedure. However, its effect in the treatment of atrial fibrillation in not reported uniformly and in large number of patients. Therefore, our aim of study was to assess the impact of concomitant radiofrequency ablation in the treatment of atrial fibrillation during cardiac surgery. We performed literature review on PubMed Central to evaluate effect of concomitant radiofrequency ablation for atrial fibrillation treatment. About 303 papers were found using the reported search, of which 15 represented suitable to fulfill our query. The authors, date, patient group, study type, relevant outcomes and results of these papers are tabulated. We conclude that radiofrequency ablation surgery of left atrium at the time of other cardiac procedures is a comparatively straight forward procedure with satisfactory freedom from atrial fibrillation, acceptable morbidity, mortality, and minor procedure related complications. Careful patients selection by sticking to the "Rule of 5", i.e. left atrial diameter less than $55 \mathrm{~mm}$ and atrial fibrillation duration no more than five years, is recommended to optimize the result of atrial fibrillation surgery.
\end{abstract}

Key words: Atrial fibrillation; Radiofrequency ablation; Surgery.

DOI: https://doi.org/10.3126/jkmc.v8i2.28174

\section{INTRODUCTION}

Atrial fibrillation (AF) is the most common type of arrhythmia presenting in the clinical practice. It is estimated that 2.2 million people suffer from AF in the United States alone and that this number will increase steadily over the next few decades, exceeding 10 million by $2050^{1}$. About $40-60 \%$ of the patients undergoing mitral valve surgery will have one or either form of $\mathrm{AF}^{2,3}$. Symptoms of $\mathrm{AF}$ include palpitations, chest pain, dyspnoea and fatigue. Stroke is a frequent life threatening complication of AF accounting for $5 \%$ in non-coagulated patients every year. The risk of stroke

Address for correspondence

Dr. Kaushal K Tiwari

Associate Professor and Head, Department of CTVS

Chitwan Medical College, Bharatpur-10, Chitwan, Nepal

E-mail: drkaushalkt@yahoo.com increases substantially with age, from $1.5 \%$ in individual ages $50-59$ years to $23.5 \%$ for those aged $80-89$ years ${ }^{4,5}$. Medical treatment and electric conversion are the first line treatment options. However, medical therapy has its limitation with failure rates as high as $60 \%$. Furthermore, during cardiac surgery it is frequently encountered as a concomitant disease in patients undergoing surgery for other reason ${ }^{2}$. In patients with permanent and persistent $\mathrm{AF}$ and large left atrium, mitral valve surgery alone is associated with a low rate of conversion to sinus rhythm during early and long-term follow-up ${ }^{6}$. Considering all these data and the high burden of AF in the general population led to search for a better treatment option by developing interventional and surgical method of treatment. 
Cox-Maze III procedure proposed by Prof James Cox became a gold standard for the surgical treatment of AF with an efficacy of $97 \%$ freedom from symptomatic $\mathrm{AF}^{7}$. However, use of Cox-Maze procedure was restricted due to its complexity, invasiveness and postoperative complications ${ }^{8}$. Cox-Maze IV, a modified version of original Cox-Maze procedure using radiofrequency and cryo energy was proposed to simplify and shorten the surgical procedure ${ }^{9}$. The decision to perform concomitant radiofrequency ablation for AF during open cardiac surgery depends mainly on a risk/benefit assessment of the procedure and the ambition of the individual patient ${ }^{10}$. Furthermore, effect of RF ablation during cardiac surgery is not reported uniformly as well as in a large series of patient populations.

In this scenario, aim of our study is to review the role of concomitant RF ablation during cardiac surgery for the treatment of AF. Additionally, to establish clearly results of such treatment in terms of freedom from arrhythmia and mortality during long term follow up.

\section{METHODS}

We performed a literature review to search for evidence of concomitant radiofrequency ablation (RFA) therapy in patients undergoing cardiac surgeries for other reasons. The search was performed in the PubMed interface from 1950 till May 2018 for English language literatures using following terms: atrial fibrillation, concomitant RF surgical ablation.

\section{RESULTS}

303 papers were found using the reported search. From these 15 papers were identified based on their relevance in regards to higher number of patients enrolled, longer follow-up time and study design (prospective randomized control trials were preferred to retrospective series). All the articles were evaluated first by studying the abstracts. Most relevant studies were selected and distributed among the authors to evaluate their strength and quality of publication. After discussion among the authors, ultimately fifteen studies fulfilling our selection criteria were selected to be included in the review. We have excluded non randomized studies, studies with small number of patients, case reports, short communication and paper published in other than English language journals. If articles from same author were repeated, most recent publication from that author was selected as most of the repeated articles are continuation of the previous study with more number of patients. Selected papers are presented in table 1.

\section{DISCUSSION}

In 2011, Geidel and coauthors ${ }^{10}$ studied 325 patients undergoing isolated mitral valve surgery $(n=201)$, Coronary Artery Bypass Grafting (CABG) and/or Aortic Valve Replacement (AVR) $(n=124)$ and concomitant radiofrequency ablation. All patients had persistent AF. The lesion set include bipolar RF pulmonary vein isolation using AtriCure device (Articurelnc, Cincinnati, $\mathrm{OH})$ plus two connecting lesions using monopolar Cobra device (Boston Scientific Corp, San Jose, Calif). Three year survival was $94.4 \%$ with freedom from $\mathrm{AF} 72.1 \%$ at discharge and $75.6 \%$ at 3 year follow-up. Moreover, in the subgroup of patients undergoing only CABG and AVR surgery conversion to stable sinus rhythm was better, $78.3 \%$ and $81.8 \%$ respectively, probably due to small Left atrium (LA) size and shorter duration of AF. In fact, they found using univariate logistic regression analysis, preoperative LA size (less than $55 \mathrm{~mm}$ ) and duration of atrial fibrillation (less than 5 years) as independent factors for successful ablation surgery. Cut off value of $55 \mathrm{~mm}$ LA size and AF duration of less than 5 years were correlated to stable SR at follow-up. Patients in stable sinus rhythm showed a significant improvement in post operative NYHA functional class (from $3.1 \pm 0.4$ to $1.7 \pm 0.4 ; p<.0001)$.

Halkos et al. ${ }^{11}$ retrospectively analyzed 54 patients undergoing left atrial (42 patients) or biatrial (12 patients) monopolar RF ablation using Cardioblate probe (Medtronic, Minneapolis, MN). Seventy percent of patients were free from AF at discharge, while $77.3 \%$ were free from AF at a median follow up about 9 months. In spite of the low statistical power related to the small population, the authors suggested that isolated mitral valve surgery with RF ablation had better freedom from $\mathrm{AF}$ rate than other procedures plus RF ablation $(88.0 \%$ vs $63.2 \% ; p=0.07$ ).

In a prospective cohort study, Beukema \& colleagues $^{12}$ enrolled 258 patients, with longstanding persistent AF having at least 12 months duration, for LA radiofrequency ablation during surgery for structural heart disease. In their cohort of patients, 3.9\% patients died during hospital stay, whereas $28.2 \%$ mortality was observed during the follow-up of 43 months. At a follow-up of 9 years they observed conversion to stable sinus rhythm in $57 \%$ patients. In support of other studies, authors have also found preoperative LA size, duration of AF and F wave amplitude as a prognostic factor for recurrence of AF or sustained AF. Ironically, they have concluded that conversion to sinus rhythm is not related to reduction in mortality and stroke rate. 


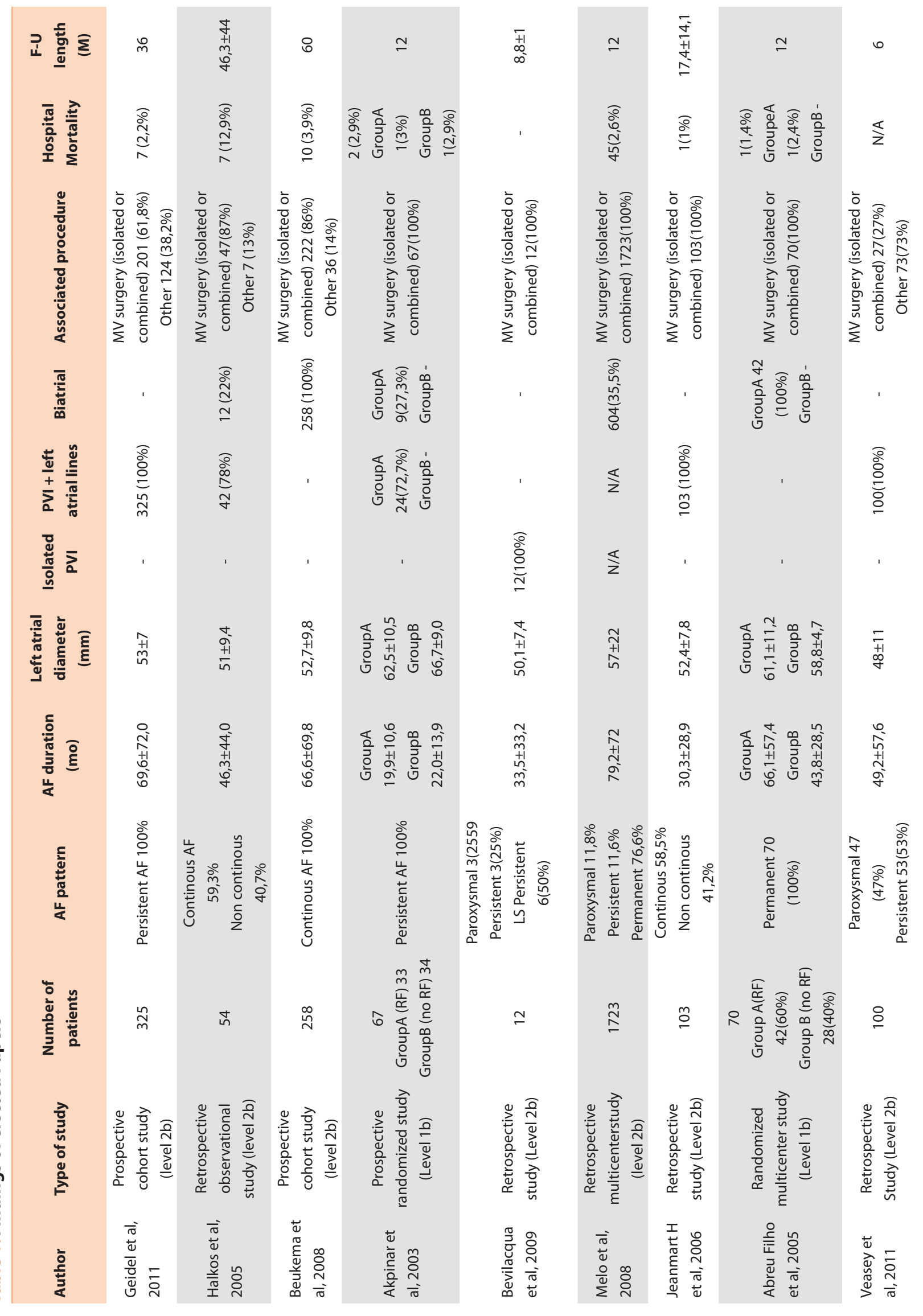




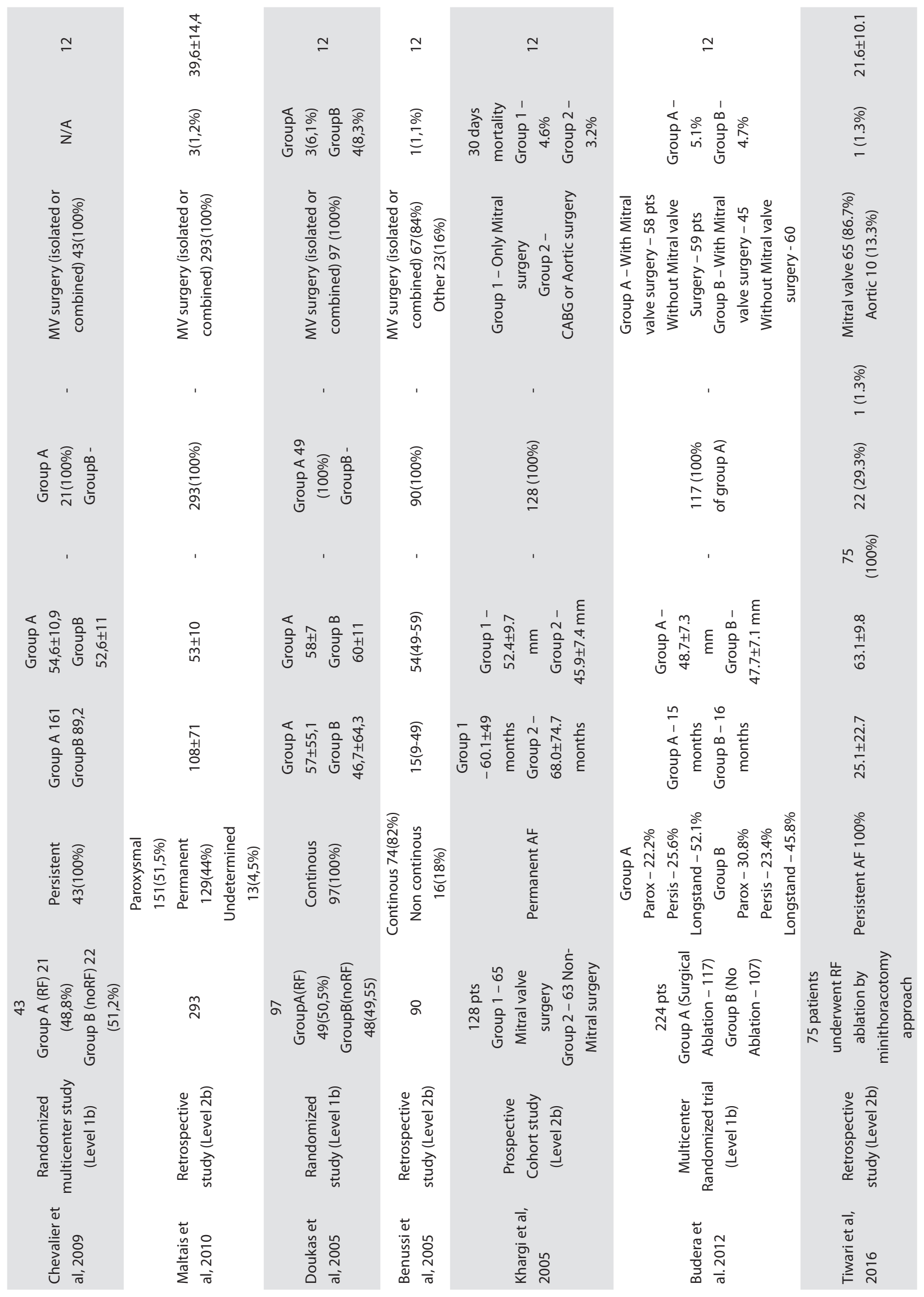


In a study by Khargi et al. ${ }^{13}$ evaluated role of antiarrythmic procedure for permanent AF in a group of patients undergoing mitral valve surgery and another group of patients undergoing non-mitral valve surgery, i.e. CABG and/or Aortic valve surgery using unipolar RFA. They have achieved a cumulative postoperative SR in $71 \%$ and $79 \%$ of patients in Group 1 and Group 2 respectively. They conclude that RFA during CABG and Aortic valve surgery is as effective as in Mitral valve surgery.

Furthermore, in prospective randomized study, Akpinar et al. ${ }^{14}$ studied chronic AF patients undergoing minithoracotomy port access mitral valve surgery. The study included 33 patients (Group A) undergoing mitral valve surgery combined with irrigated RF ablation using monopolar ablation device, while 34 patients undergoing only mitral valve surgery as control group (Group B). At the discharge $81 \%$ of the group A patients were free of $\mathrm{AF}$, whereas only $15.2 \%$ of the patients in group B. Most importantly, at a follow-up of 1 year most of the patients in group A (93.6\%) were free from AF vs. 9.4\% in group B. Anti-arrhythmic drugs were discontinued earlier in group A patients (3 months vs. 1 year).

In our own experience of concomitant RF ablation for AF during minimally invasive port access mitral valve surgery using an epicardial vacuum assisted monopolar Cobra Adhere XL probe (Estech, San Ramon, CA), we observed $91.7 \%$ freedom from AF at a follow up of 9 months. No major cardiac and cerebro-vascular events occurred during follow-up ${ }^{15}$. We have also observed better cosmetic result with less post operative pain in the patients treated with this approach.

Melo and colleagues ${ }^{16}$ reported a multicenter study enrolling 1723 patients, with different type of AF and mean AF duration of $6.6 \pm 6$ years, from 10 European and American hospitals in a period of three years. Three percent of the patients underwent cut-and-sew surgery, while $97 \%$ underwent ablation with different types of energy. $65 \%$ of the patients were ablated with unipolar and bipolar RF energy, 27\% microwave, 4\% Argon cryoablation and $4 \%$ other type of energy. Fifty nine percentages of the patients were in sinus rhythm at the discharge, while $66 \%$ were in stable sinus rhythm (sSR) after one year of follow-up. Before discharge 3\% of patients required pacemaker implantation. On log rank analysis they found that stable SR was associated with higher early and late survival $(p=.01)$ and lower thromboembolic events $(p=.010)$. Left atrial dimension $(>55 \mathrm{~mm})$ and concomitant coronary revascularization was found to be independent negative predictor of
sSR. Use of cut-and-sew technique and isolated left atrial procedure for AF treatment were reported to be independent predictors for in-hospital mortality.

Jeanmart et al. ${ }^{17}$ studied hundred and three patients with intermittent (41.2\%) and continuous (58.8\%) $\mathrm{AF}$, undergoing minimally invasive mitral valve surgery and concomitant pulmonary veins isolation with monopolar radiofrequency using Cardioblate ${ }^{\circledR}$ irrigated RF ablation probe (Medtronic, Inc, USA). At a follow-up of 18 months $69.7 \%$ of patients were in sinus rhythm, while $28.3 \%$ were in atrial fibrillation, $2 \%$ patients were pacemaker dependent, and $81.2 \%$ of treated were still taking antiarrhythmic drugs (AAD) in comparison to $76.5 \%$ on AAD at discharge.

Seventy patients with permanent AF lasting for more than 1 year and rheumatic mitral valve disease were randomized by Abreu Filho and colleagues ${ }^{18}$ in two groups. The RF ablation group ( $n=42$ patients) underwent RF ablation of right and left atrium using monopolar probe plus mitral valve surgery, while the control group underwent only mitral valve surgery. There was no in-hospital mortality in the control group, whereas one death (2.3\%) was in RFA group, which was not related to ablation procedure. A significant difference in conversion to SR among the two groups at the time of discharge (75.6\% in RFA group vs. $17.9 \%$ in control group) as well as at the end of 12 months followup $(79.4 \%$ vs. $26.9 \%)$ was evident.

For the first time, Veasey et al $^{19}$, evaluated heart rhythm for 7 days by Holter monitoring in one hundred patients with paroxysmal and persistent AF undergoing RF ablation of left atrium at the time of concomitant cardiac surgery. Pulmonary veins were isolated as pairs with a connecting line to the left atrial appendage using Articure bipolar RF probe (Articurelnc, Cincinnati, OH). In their cohort of patients, they observed slight decrease in freedom from AF at 12 months follow-up, from 86\% at the discharge to $62 \%$ at the follow-up. However, they have found a reduction of AF burden from $56.2 \%$ to $27.5 \%$ in patients with RF ablation. In addition, they also confirmed the duration of AF, persistent AF and mitral valve surgery as significant predictors of AF recurrence.

A multicenter, double-blinded randomized study conducted by Chevalier et $\mathrm{al}^{20}$, enrolled 43 patients with persistent AF for more than 6 months either to undergo RF ablation plus mitral valve surgery or mitral valve surgery alone. They isolated pulmonary veins and created additional lesions toward the mitral annulus. There was a significant difference in conversion to sinus 
rhythm at discharge $(72.73 \%$ in RFA group vs. $4.76 \%$ in control group). This trend of significance remained constant at the end of follow-up. Although pacemaker implantation were frequent in RFA group (14.3\% vs. $9.1 \%)$, need of electric cardioversion at 12 months follow-up was less in RFA group (9.52\% vs. 50\%).

Another important and large multicentre RCT, PRAGUE12 ,by Budera et a $^{21}$ has divided overall 224 patients in two groups. Group A undergoing cardiac surgery with RFA and Group B without ablation. In group A patients they have created a complete lesion of ablation. Authors have analyzed ECGs in regular follow up of 1, 3, 6 and 2 months. At a follow up of 1 year, $60.3 \%$ of group A and $35.5 \%$ of group B patients were in SR. Additionally, they have observed a remarkable, but non-significant, difference in stroke rate in favor of ablated patients at 1 year follow up.

Maltais and co-authors ${ }^{22}$ retrospectively studied results of 293 patients, who underwent cardiac surgery with concomitant RF ablation of left atrium using monopolar probe. They observed a mortality of $1.2 \%$ not related to RF ablation procedure. Furthermore, authors also noticed a trend in increasing conversion to sinus rhythm at the end of 40 months follow-up (52\% vs. $71 \%$ at follow-up).

Doukas and colleagues ${ }^{23}$ had studied 97 patients with continuous AF for at least 6 months, who were undergoing surgery for mitral valve plus left atrium RF ablation and mitral valve surgery only. In their randomized study, in hospital mortality was not different in both groups. Conversion to sinus rhythm at discharge $(53.3 \%$ vs. $4.5 \%)$ and at a 12 months followup ( $44.5 \%$ vs. $4.5 \%, \mathrm{P}<.001)$ was significantly higher in $\mathrm{RF}$ ablation group in compared to mitral valve surgery only. Furthermore, authors have stressed on the importance of conversion to SR, which had significantly greater improvement in Shuttle-walk test.

In a cohort of 90 patients with continuous and intermittent AF, Benussi and coauthors ${ }^{24}$ has shown $89 \%$ freedom from $A F$ at one year follow-up using a bipolar complete left atrial RF ablation. Moreover, they emphasize that use of bipolar RF grants acute transmurality of the lesionswith no major complications related to the AF procedure. Furthermore, they advise addition of an extra lesion to the mitral annulus to prevent left atrial flutter.

Overall freedom from AF in the reported studies using RF ablation during concomitant cardiac surgery is satisfactory at the discharge, although highly variable. A trend towards higher freedom from AF at the end of follow up period was observed in various studies. It could be due to a transient phase of pro-arrhythmic state in the early postoperative period. Importantly, symptomatic relief in the patients treated with RF ablation was evident, including improved shuttle-walk test. In addition, some authors reported decrease in use of anti-arrhythmic drugs after the ablation, which could contribute to a better outcome in terms of patient's survival. Besides, in all randomized study it has been well demonstrated a net benefit of RF ablation in terms of SR restoration in patients with concomitant AF surgery, which is associated with clinically symptom's improvement, decrease in thromboembolic events and better functional outcome. No mortality was directly related to the AF ablation procedure. However, RF ablation treatment has not demonstrated any superiority in term of mortality reduction in AF patients. But, conversion to stable SR is associated with higher early and late survival.

Minimally invasive approaches for RF ablation have been used in few studies. In our own study, we have performed ablation in 75 for $\mathrm{AF}$ in patients undergoing different valvular surgeries through right sided minithoracotomy. All of the patients were ablated with radiofrequency energy. In the majority of cases, i.e. in $82.7 \%$ of cases, monopolar energy was implemented. After a mean follow-up of $21.6 \pm 10.1$ months (range: 5 - 45 months), $98.6 \%$ of patients completed the follow up, $46(63 \%)$ were in a stable sinus rhythm, and 27 (37\%) remained in AF. However, out of 17 patients with paroxysmal AF, 14 (82.4\%) converted into SR. While $70 \%$ of patients with persistence AF converted into SR. We observed minithoracotomy approach to be as safe as conventional sternotomy, with better cosmetics and less pain ${ }^{25}$. Further studies with larger population might need to draw a conclusion about the use of minimal invasive approach.

\section{LIMITATIONS OF REPORTED STUDIES}

Before making any conclusion, we must underline obvious limitations of studies being performed. First of all, in several studies low statistical power due to the small sample size was evident. With the relentless evolution and advancement of new technology for ablation, different type of devices and lesion set were used throughout these studies. Furthermore, selection criteria, definition of AF, end points (freedom from $A F$, freedom from symptomatic or asymptomatic $\mathrm{AF}$ ), $\mathrm{AF}$ burden, follow-up criteria (duration, and method of ECG registration), use of anti-arrhythmic drugs and its 
duration were not standardized. Such non-standardized evaluation of the results generated variability and difficulty in the elaboration and presentation of the outcomes. In future, a better compliance is needed to standardize definition, recommendations and evaluation criteria as proposed by HRS/EHRA/ECAS expert consensus conference on catheter and surgical ablation of $\mathrm{AF}^{26}$.

\section{CONCLUSION}

On the basis of our review, we conclude that overall freedom from $A F$ in patients undergoing concomitant $\mathrm{RF}$ ablation during other cardiac procedures is satisfactory. Restoration of sinus rhythm after RF ablation is associated with symptomatic relief and improved functional capacity of treated patients as well as decreased use of anti-arrhythmic drugs in post operative period. Importantly, concomitant RF ablation is not associated with higher mortality.

Finally, "Rule of 5", i.e. a cut off value for left atrial diameter less than $55 \mathrm{~mm}$ and AF duration less than 5 years, could be implemented in clinical practice to achieve better result in terms of freedom from $A F$ and stable sinus rhythm at the long term follow-up.

Damiano RJ Jr. A prospective, single-center clinical trail of a modified Cox Maze procedure with bipolar radiofrequency ablation. J Thorac Cardiovasc Surg 2004;128:535-542. [FullText]

10. Geidel S, Krause K, Boczor S, Kuck KH, Lass M, Ostermeyer J, Schmoeckel M. Ablation surgery in patients with persistent atrial fibrillation: An 8-year clinical experience. J Thorac Carciovasc Surg 2011;141:377-82. [DOI]

11. Halkos ME, Craver JM, Thourani VH, Kerendi F, Puskas JD, Cooper WA, Guyton RA. Intraoperative radiofrequency ablation for the treatment of atrial fibrillation during concomitant cardiac surgery. Ann ThoracSurg 2005;80:210-6. [PubMed]

12. Beukema WP, Sie HT, Misier ARR, Delnoy PPHM, Wellens HJJ, Elvan A. Intermediate to long-term results of radiofrequency modified Maze procedure as an adjunct to open-heart surgery. Ann Thorac Surg 2008;86:1409-14. [DOI]

13. Khargi K, Lemke B, Deneke T. Concomitant antiarrhythmic procedures to treat permanent atrial fibrillation in CABG and AVR patients are as effective as in mitral valve patients. Eur J Cardiothorac Surg 2005; 27: 8416. [DOI]

14. Akpinar B, Guden M, Sagbas E, Sanisoglu I, Ozbek U, Caynak B, Bayindir O. Combined radiofrequency modified maze and mitral valve procedure through a port access approach: early and mid-term results. Eur J Cardiothorac Surg 2003;24:223-30. [DOI]

15. Bevilaqua S, Gasbarri T, Cerillo AG, Mariani M, Murzi M, Nannini T, Glauber M. A new vacuum assisted probe for minimally invasive radiofrequency ablation. Ann Thorac Surg 2009;88:1317-21. [DOI]

16. Melo J, Santiago T, Aguiar C, Berglin E, Knaut M, Alfieri $\mathrm{O}$, Benussi S, Sie H, Williams M, Hornero F, Marinelli G, Ridley P, Fulquet-Carreras E, Ferreira A. Surgery for atrial fibrillation in patients with mitral valve disease: Results at five years from the International Registry 
of Atrial Fibrillation Surgery. J Thorac Cardiovasc Surg 2008; 135:863-9. [DOI]

17. Jeanmart $H$, Casselman F, Beelen R, Wellens F, Bakir I, Van Praet F, Cammu G, Degriek Y, Vermeulen Y, Vanermen $\mathrm{H}$. Modified Maze during endoscopic mitral valve surgery: The OLV clinic Experience. Ann Thorac Surg 2006; 82:1765-9. [DOI]

18. Abreu Filho CAC, Lisboa LAF, Dallan LAO, Spina GS, Grinberg M, Scanavacca M, Sosa EA, Ramires JAF, Oliveira SA. Effectiveness of Maze procedure using cooled-tip radiofrequency ablation in patients with permanent atrial fibrillation and rheumatic mitral valve disease. Circulation 2005;112[suppl I]:-20 I-25. [DOI]

19. Veasey RA, Segal OR, Large JK, Lewsi ME, Trivedi UH, Cohen AS, Hyde JA, Silke An. The efficacy of intraoperative atrial radiofrequency ablation for atrial fribrillation during concomitant cardiac surgery-the Surgical Atrial Fibrillation Supression (SAFS) study. J Interv Card Electrophysiol 2001;32:29-35. [DOI]

20. Chevalier P, Leizorovicz A, Maureira P, Carteaux JP, Corbineau H, Caus T, DeBreyne B, Mabot P, Dechillou C, Deharo JC, Barry S, Touboul P, Villemot JP, Obadia JF. Left atrial radiofrequency ablation during mitral valve surgery: a prospective randomized multicenter study (SAFIR). Archives of cardiovascular disease 2009;102:769-75. [DOI]

21. Budera $P$, Straka $Z$, Osmaněík $P$, Vaněk $T$, Jelínek $S$, Hlavička J, Fojt R, Červinka P, Hulman M, Šmíd M, Malý M, Widimský P. Comparison of cardiac surgery with left atrial surgical ablation vs. cardiac surgery without atrial ablation in patients with coronary and/ or valvular heart disease plus atrial fibrillation: final results of the PRAGUE12 randomized multicentre study. Eur Heart J2012; 33: 264452. [DOI]

22 Maltais S, Forcillo J, Bouchard D, Carrier M, Cartier R, Demers $P$, Perrault LP, Poirier N, Ladouceur $M$, Pagè $P$, Pellerin $M$. Long-term results following concomitant radiofrequency modified Maze ablation for atrial fibrillation. J Card Surg 2010;25:603-8. [DOI]

23. Doukas G, Samani NJ, Alexiou C, Oc M, Chin DT, Stafford PG, Ng LL, Spyt TJ. Left atrial radiofrequency ablation during mitral valve surgery for continuous atrial fibrillation. A randomized controlled trial. JAMA 2005;294:2323-9. [DOI]

24. Benussi S, Nascimbene S, Calori G, Denti P, Ziskind Z, Kassem S, La Canna G, Pappone C, Alfieri O. Surgical ablation of atrial fibrillation with a novel bipolar radiofrequency device. J Thorac Cardiovasc Surg 2005;130:491-7. [DOI]

25. Tiwari KK, Gasbarri T, Bevilacqua S, Glauber M. Right sided Minithoracotomy as a surgical approach for the concomitant treatment of atrial treatment. Res Cardiovasc Med. 2016 May; 5(2): e31374. [DOI]

26. Calkins H, Brugada J, Packer D, Cappato R, Chen SA, Crijns HJG et al. HRS/EHRA/ECAS expert consensus statement on catheter and surgical ablation of atrial fibrillation:Recommendations for personnel, policy, procedure and follow-up. Europace 2007;9:335-79. [DOI] 